\title{
Instrumentos económicos basados en mercados para la conservación de la biodiversidad y los servicios ecosistémicos en Latinoamérica: ¿panacea o rueda cuadrada?
}

\author{
Sebastián Aguiar ${ }^{1, \otimes}$; Gonzalo Camba Sans ${ }^{1} \&$ José M. Paruelo ${ }^{1 / 2}$ \\ ${ }^{1}$ Laboratorio de Análisis Regional y Teledetección. Facultad de Agronomía, Universidad de Buenos Aires. IFEVA, CONICET. \\ Argentina. ${ }^{2}$ Instituto de Ecología y Ciencias Ambientales. Facultad de Ciencias. UdelaR. Uruguay.
}

\begin{abstract}
Resumen. La percepción de que las estrategias que llevan adelante los gobiernos para asegurar la conservación de la biodiversidad y la provisión de servicios ecosistémicos son ineficaces o insuficientes dieron lugar a nuevas alternativas basadas en instrumentos económicos, impulsadas tanto por actores privados (en particular, ONG) como por los mismos gobiernos. Algunos de estos instrumentos buscan internalizar una externalidad positiva al compensar al proveedor del servicio ecosistémico (SE) por los costos en los que incurre al asegurar su provisión. Actualmente, no existe consenso respecto a las ventajas y desventajas de los instrumentos económicos basados en mercados para la conservación (IEBMC), y la mayor parte de este debate ha ocurrido en el plano teórico. Esto hace imprescindible la necesidad de evaluaciones empíricas. El objetivo de este trabajo fue caracterizar 60 proyectos que incluyeran IEBMC en Latinoamérica en cuanto a su efectividad y equidad social, y la relación de éstas con diversas características económicas, políticas e institucionales de los proyectos. A través de ello buscamos explorar si existen sinergias o compromisos entre objetivos ambientales y sociales, y si hay características que determinan que los proyectos sean más exitosos respecto a estos objetivos. El 43\% de los proyectos resultaron efectivos en cuanto a sus objetivos ambientales, mientras que $\sim 17 \%$ resultaron equitativos. La relación entre efectividad y equidad no resultó estadísticamente significativa, lo que indica que no hay sinergias ni compromisos entre ellas. En general, tanto la efectividad como la equidad no se asociaron de manera significativa con ninguna de las características relevadas. Esto implica que no hay un único arreglo institucional que asegure el buen desempeño de este tipo de mecanismos. Nuestros resultados sugieren que no existen evidencias suficientes de que los IEBMC sean ventajosos para conservar la biodiversidad y los SE de forma efectiva y socialmente equitativa en Latinoamérica.
\end{abstract}

[Palabras clave: pago por servicios ecosistémicos, PSA, servicios ambientales, equidad, efectividad, Sudamérica, Centroamérica]

\begin{abstract}
Aвstract. Market-based instruments for the conservation of biodiversity and ecosystem services in Latin America: panacea or square wheel? The perception that the strategies carried out by governments for biodiversity conservation and ecosystem services provision are ineffective or insufficient, led to diverse new alternatives based on economic instruments driven by both private actors (fundamentally NGO's) and governments. Some of these instruments seek to internalize a positive externality by compensating the ecosystem service (ES) provider for the costs incurred in ensuring its provision. Currently, there is no consensus on the strengths and weaknesses of the market based economic instruments for nature conservation (MBEINC), and most of this debate has taken place in a theoretical arena. Thus, the need for empirical assessment is essential. The objective of this work was to characterize 60 projects, which included MBEINC, in Latin America in terms of their effectiveness and social equity, and the relation of them with diverse economic, political and institutional characteristics. Through this we seek to explore whether there are synergies or tradeoffs between environmental and social objectives, and determine which characteristics determine that the projects are more successful with respect to these objectives. $43 \%$ of the projects were effective in terms of their environmental objectives, while approximately $17 \%$ were socially equitable. The relationship between effectiveness and social equity was not statistically significant, indicating that there are no synergies or tradeoffs between them. In general, both effectiveness and social equity were not significantly associated with any of the characteristics surveyed. This implies that there is no single institutional arrangement to ensure the positive outcomes of this type of mechanism. Our results suggest that there is insufficient evidence of the advantages of MBEINC to conserve biodiversity and ES in an effective and socially equitable manner in Latin America.
\end{abstract}

[Palabras clave: payment for ecosystem services, equity, effectiveness, South America, Central America, PES]

Editora asociada: Berta Martín López

$\triangle$ aguiarse@agro.uba.ar 


\section{INTRODUCCIÓN}

Los servicios ecosistémicos (SE) (i.e., los beneficios que los humanos obtienen de la naturaleza [MEA 2005]) se concibieron en principio como una metáfora y luego se convirtieron en un marco conceptual para estudiar y hacer explícita la dependencia del bienestar humano de los ecosistemas (Norgaard 2010). Desde 2005, con el Millenium Ecosystem Assessment (MEA 2005), los SE han sido el paradigma principal en el que se enmarca la investigación y las políticas públicas y privadas, orientadas a la conservación y restauración de los ecosistemas (Silvertown 2015). Este paradigma resulta de una modificación en la forma en que se analiza la relación entre el hombre y la naturaleza, cuya última etapa puede caracterizarse, entre otras cosas, por una tendencia a la valoración monetaria de la naturaleza y a la resolución de los problemas ambientales con una aproximación económica (Mace 2014). En parte, esto podría deberse a que ciertos actores creen que las medidas que toman los gobiernos para asegurar la conservación de la naturaleza (e.g., a través de leyes ambientales o de la creación de áreas protegidas) son insuficientes o ineficaces (Gaston et al. 2008; Mascia and Pailler 2011). En este contexto, en el siglo XXI se multiplicaron las alternativas asociadas a actores privados tales como ONG y diferentes tipos de corporaciones (e.g., empresas, bancos) como complementos de la acción estatal para asegurar la conservación (Kareiva and Marvier 2012; Moreno-Sánchez 2012; Galaz et al. 2015). Esta tendencia dio lugar a una polarización en el ámbito académico respecto a los instrumentos económicos para la conservación, tales como los pagos por servicios ecosistémicos (PSE) (Tacconi 2012; Muradian et al. 2013; Wunder 2013). Sin embargo, la mayor parte de este debate tuvo lugar en el plano teórico e ideológico, con poca evidencia empírica sobre la aplicación concreta de estos instrumentos.

Los instrumentos económicos para la conservación de la naturaleza son una herramienta de política ambiental cuyo objetivo es proveer incentivos para reducir la degradación ambiental (Azqueta et al. 2007). A diferencia de otras políticas, tales como las normativas o las leyes, el agente puede decidir degradar el ambiente y pagar un precio por ello (principio de "contaminador paga"), o no hacerlo y ser compensado económicamente (principio de "proveedor obtiene" (Azqueta et al. 2007). Estos instrumentos son diversos y difíciles de definir y clasificar (Pirard and Lapeyre 2012; Vatn 2015). A grandes rasgos, incluyen mecanismos que modifican el comportamiento de los agentes vía precios (e.g., impuestos, subsidios) o crean mercados en los que existe una compensación a los proveedores del SE (Moreno-Sánchez 2012). Dos de las formas más difundidas de estos últimos son los PSE (Engel et al. 2008) y las compensaciones por biodiversidad (Bull et al. 2013; Ives and Bekessy 2015). La lógica común que subyace a estos instrumentos está basada sobre el concepto de externalidad, a la que desde la economía neoclásica se la considera una falla de los mercados. Las externalidades pueden ser tanto positivas como negativas y surgen por la inexistencia de un valor de mercado para determinados bienes y servicios. Esto, en última instancia, implica que no sean considerados en la distribución de beneficios y perjuicios entre los agentes económicos (Gómez-Baggethun and Muradian 2015). Por lo general, estos mecanismos incluyen una compensación (no necesariamente monetaria) al proveedor o generador de la externalidad positiva, por los costos (comúnmente de oportunidad, aunque no siempre surgen de una evaluación explícita) en los que incurre para garantizar la provisión del servicio (Engel et al. 2008). Es decir, la compensación es la forma de internalizar la externalidad positiva.

La expansión de los instrumentos económicos basados en mercados para la conservación (IEBMC) es innegable (Pirard 2014) y está determinando cambios clave en la gobernanza ambiental (Vatn 2010). Algunos académicos argumentan que los IEBMC son superiores respecto a las alternativas estatales pues no sólo son más eficientes en sentido económico (Engel et al. 2008; Kinzig et al. 2011), sino que también crean situaciones virtuosas para aliviar la pobreza rural (Pagiola et al. 2005) y promover la innovación tecnológica (Lockie 2013). Sin embargo, el crecimiento acelerado de los IEBMC no está exento de críticas, y para algunos es un ejemplo más del avance del mercado sobre dominios tradicionalmente gobernados por otro tipo de normas e instituciones (Harvey 2007; Sandel 2012; Silvertown 2015). Las críticas a los IEBMC son múltiples; provienen de diferentes disciplinas y marcos conceptuales y están acompañadas de contraargumentos (Schröter et al. 2014; Gómez-Baggethun and Muradian 2015). Las críticas suelen centrarse en alguna de las tres etapas del proceso de mercantilización ('commodification' en inglés) de los SE 
(Kosoy and Corbera 2010). Estas etapas son: a) enmarcar una función ecológica como un servicio que satisface necesidades humanas, b) asignarle a este servicio un único valor de cambio, y c) conectar a los proveedores y usuarios de los servicios en un mercado. En definitiva, no existe consenso respecto a los beneficios y a los perjuicios de los IEBMC (ver Muradian et al. 2013 y las respuestas por Wunder 2013), y la mayor parte del debate ha tenido lugar en el plano teórico respecto a su concepción como instrumento de gestión, más que en su implementación.

El desempeño de los IEBMC puede medirse en diferentes dimensiones, tales como efectividad, eficiencia, equidad, flexibilidad y legitimidad (Adger et al. 2005; Azqueta et al. 2007). En general, la evaluación del desempeño ha estado centrada en la efectividad, y en particular sobre la eficiencia; es decir, asegurar la provisión del SE al menor costo posible (Pascual et al. 2014). Esto puede ser problemático debido a que resultados eficientes pueden modificar y profundizar las inequidades existentes entre los actores participantes (Corbera et al. 2007). Por ello, en la actualidad, la evaluación del desempeño se amplió para incluir aspectos distributivos relacionados con la equidad social entre los actoresqueparticipandela transacción(Pascual 2014). Por esta razón, la evaluación conjunta de ambas dimensiones (efectividad ambiental y equidad social) permite describir con más detalle el desempeño de estos instrumentos, pues los aspectos distributivos podrían afectar el desempeño ambiental de los proyectos. La relación entre ambas dimensiones puede ser sinérgica (cumplimiento de ambos tipos de objetivos), de compromiso (cumplimiento de un tipo de objetivo, pero no del otro) o de independencia (objetivos sociales y ambientales no se relacionan). Que sea de un tipo u otro depende de varios condicionantes (Pascual et al. 2010). Por ejemplo, considerar cuestiones de equidad social en el diseño puede tener implicancias fuertes en la legitimidad de las intervenciones, y ello podría aumentar la probabilidad de obtener buenos resultados ambientales (Pascual et al. 2010). En este contexto, algunos autores sugieren que no existen razones por las cuales la eficiencia deba prevalecer sobre otros objetivos sociales, tales como la equidad, a la hora de diseñar e implementar políticas ambientales (Muradian et al. 2010), y que su ponderación es una cuestión normativa (Pascual et al. 2012).

Latinoamérica (LA) ha sido pionera en la implementación de IEBMC y es una de las regiones del mundo con mayor cantidad de programas en ejecución (Wunder et al. 2005; Ezzine-de-Blas et al. 2016). En varios trabajos publicados recientemente se evaluaron diversas características de estos instrumentos (e.g., efectividad y equidad), lo cual permitió comprender con mayor detalle la implementación deestosinstrumentos. MartínOrtega (2010) analizaron 40 proyectos de PSE en LA relacionados con la provisión de agua y encontraron, entre otras cosas, que hay una gran divergencia entre el empleo de este tipo de instrumentos y lo que la teoría indica que son. Para la misma región, Grima et al. (2014) analizaron 40 proyectos de PSE. Encontraron que el cumplimiento de los objetivos ambientales está relacionado con diferentes características de los programas, aunque sus inferencias no se basan en análisis estadísticos. Por ejemplo, encontraron que los proyectos sin intermediarios, de larga duración y con el SE bien definido tienden a ser más exitosos. Para 29 programas ejecutados en países tropicales (19 en LA), Calvet et al. (2015) analizaron la efectividad y la equidad, y encontraron que la efectividad tiende a ser mayor a la equidad y que ésta a menudo presenta resultados negativos. Ezzine-de-Blas et al. (2016) analizaron 55 programas distribuidos globalmente y hallaron que aquellos programas que segmentan espacialmente con mayor precisión la provisión de SE, o su amenaza, tienden a ser más efectivos. La mayoría de estos estudios se basan sólo en información publicada en artículos científicos, lo que reduce la cantidad de casos a describir y omite gran cantidad de información publicada en informes gubernamentales y otros tipos de publicaciones sin referato.

El objetivo de este trabajo es caracterizar proyectos que incluyeran IEBMC en LA en cuanto a su efectividad y equidad social, y la relación de ellas con diversas características económicas, políticas e institucionales de los instrumentos. A través de ello buscamos determinar si hay sinergias o compromisos entre objetivos ambientales y sociales, y si existen características que determinen que los proyectos sean más exitosos respecto a estos objetivos. El análisis recopila proyectos incluidos en bases de datos de acceso público y publicados en trabajos previos. La descripción de los proyectos se basa principalmente en Sattler et al. (2013), quienes proponen una lista exhaustiva de atributos que permite simplificar en relativamente pocas categorías el funcionamiento de estos instrumentos complejos. A diferencia de otros trabajos, se 
incluye también información publicada fuera de la literatura científica porque creemos que los tomadores de decisiones consultan informes técnicos de ONG y gobiernos a la hora de decidir sus acciones.

\section{Materiales y Métodos}

\section{Base datos}

Se conformó una base de datos con 60 proyectos que incluyen algún IEBMC en LA, únicamente para ecosistemas terrestres y de aguas continentales. Para ello se recopilaron casos publicados en diferentes bases de datos de acceso libre disponibles en internet ( $w w$ w.watershedmarkets.org/casestudies.html, www.teebweb.org/resources/case-studies/,w ww.ecosystemmarketplace.com/, ww.oas.org/ dsd/PES/Database.htm\#,www.watershedconn ect.com/programs/index.php) y en artículos científicos que incluyen proyectos ejecutados en LA (Wunder et al. 2008; Brouwer et al. 2011; Martin-Ortega et al. 2013; Schomers and Matzdorf 2013; Calvet-Mir et al. 2015; Ives and Bekessy 2015; Grima et al. 2016). Para cada caso se hicieron búsquedas de información más exhaustivas para el período comprendido entre 2000 y 2015, utilizando Scopus, Google y Google Académico.

El requisito para incluir los proyectos relevados en la base de datos es que debían estar ya ejecutados o en ejecución, ser de carácter voluntario y tener una descripción de los actores participantes de la transacción (i.e., usuarios, proveedores, intermediarios) y del funcionamiento del programa. No existe consenso en cuanto a la clasificación de los IEBMC, principalmente en cuándo y en qué medida se basan (o no) en mercados (e.g. mercados incompletos), pues en muchos casos se asemejan más a impuestos o a subsidios administrados por el Estado (Pirard 2012; Vatn 2015). Más allá de esta discusión, utilizamos la clasificación que propuso Vatn (2015), quien clasificó a los IEBMC en 4 grupos: pagos por SE, certificaciones ambientales, compensaciones por biodiversidad y mercados de carbono. En esta base de datos fueron incluidos tres de estos tipos, y se excluyeron las certificaciones ambientales.

\section{Descripción de atributos}

A cada proyecto incorporado se le asignó un número identificador, se registraron las referencias bibliográficas, la procedencia de la información (artículo académico, informe, mixto), el país de origen, el lugar en donde se implementa, y el nombre y año de comienzo. Además, se los clasificó de acuerdo con los criterios propuestos por Sattler et al. (2013) para describir proyectos de PSE. Hasta la actualidad, la propuesta de Sattler et al. (2013) para caracterizar proyectos es la más completa y exhaustiva. Sin embargo, esta clasificación está pensada y aplicada en países desarrollados, donde se dispone de más información; por esta razón, algunos de los criterios se eliminaron, otros se modificaron sutilmente y, por último, se agregaron otros no incluidos en dicha clasificación. La Tabla 1 resume y describe las características de los proyectos evaluados, y señala aquellas no incluidas en la clasificación de Sattler et al. (2013) y las que fueron modificadas respecto de lo propuesto por estos autores.

La clasificación de la efectividad y las dimensiones de la equidad se realizó en base a una interpretación de los resultados reportados en la bibliografía. Las formas de medir ambas son múltiples y tienen diferente grado de objetividad, lo cual es una limitación de este tipo de trabajos que sintetizan información de diferentes procedencias. En consecuencia, dado que la caracterización de estos criterios conlleva subjetividad, se extrajo la cita textual que fue utilizada en cada caso y se la incluyó en la base de datos (Tabla 1, material suplementario) para transparentar las decisiones y, además, se registró la metodología que se utilizó para caracterizarlas en cada caso.

La efectividad fue caracterizada respecto al cumplimiento de los objetivos ambientales propuestos por el proyecto. La adicionalidad y la condicionalidad son dos características relacionadas con la efectividad, esenciales para cierto tipo de proyectos (e.g., PSE) (Wunder 2005). La condicionalidad hace referencia a la compensación al proveedor; se considera que un proyecto presenta condicionalidad cuando la compensación se realiza sólo si el proveedor llevó a cabo las actividades que aseguran la provisión del SE. A su vez, un proyecto presenta adicionalidad cuando, en caso de no existir la compensación al proveedor, no se hubiese asegurado la provisión del SE (Tacconi 2013).

La evaluación de la equidad de los proyectos requiere analizar múltiples dimensiones (McDermott et al. 2013; Pascual et al. 2014): a) procedimiento, que se refiere a la inclusión 
Tabla 1. Caracterización de las dimensiones evaluadas en cada proyecto. Se describen los criterios utilizados para asignar las clases dentro de cada dimensión. Se utiliza $\left({ }^{*}\right)$ para indicar atributos propuestos por Sattler et al. (2013), $\left.{ }^{(* *}\right)$ para indicar atributos de Sattler et al. (2013) pero que han sido modificados y $\left.{ }^{(* *}\right)$ para atributos no descriptos por Sattler et al. (2013)

Table 1. Description of the dimensions evaluated for each project. The criteria used to assign the classes within each dimension are described. $\left(^{*}\right)$ indicates attributes proposed by Sattler et al. (2013), $\left({ }^{* *}\right)$ attributes proposed by Sattler et al. (2013) but that have been modified and $\left(^{* * *}\right)$ attributes not included by Sattler et al. (2013).

\begin{tabular}{|c|c|c|}
\hline & Clases & Descripción \\
\hline $\begin{array}{l}\text { Procedencia de la } \\
\text { información }(* * *)\end{array}$ & $\begin{array}{l}\text { Artículo científico, informe } \\
\text { y mixto }\end{array}$ & $\begin{array}{l}\text { Tipo de bibliografía consultada. Se clasificó la fuente } \\
\text { bibliográfica en las clases artículo científico, informe (literatura } \\
\text { gris) y mixto (académico e informe) }\end{array}$ \\
\hline Escala espacial $(*)$ & Local, regional y nacional & $\begin{array}{l}\text { Local para casos que abarquen hasta una cuenca y/o una } \\
\text { unidad administrativa de tercer nivel, regional para más de una } \\
\text { cuenca y/o unidades administrativas mayores al tercer nivel y } \\
\text { nacional para todo el territorio de un país }\end{array}$ \\
\hline Escala temporal $(* *)$ & Corto, medio y largo plazo & $\begin{array}{c}\text { Definida como el tiempo transcurrido entre el año de inicio del } \\
\text { proyecto y el año de su evaluación. Corto plazo para intervalos } \\
\text { menores a } 10 \text { años, medio para intervalos entre } 10 \text { y } 30 \text { años y } \\
\text { largo para mayores a } 30 \text { años }\end{array}$ \\
\hline $\begin{array}{l}\text { Servicio ecosistémico } \\
\text { (SE) }\left(^{*}\right)\end{array}$ & $\begin{array}{l}\text { Agua, carbono, } \\
\text { biodiversidad y múltiple }\end{array}$ & $\begin{array}{l}\text { SE transferido. La categoría múltiple refiere cuando hubo más } \\
\text { de un SE transferido }\end{array}$ \\
\hline $\begin{array}{l}\text { Tipo de proveedor y } \\
\text { usuario }\left(^{*}\right)\end{array}$ & Privado, público y mixto & $\begin{array}{c}\text { Sector al que pertenecen las partes de la negociación. Para } \\
\text { los compradores, además, se indicó si eran nacionales o } \\
\text { internacionales }\end{array}$ \\
\hline Intermediarios $(* *)$ & Estado, ONG y otros & $\begin{array}{l}\text { Organizadores de las transacciones. Para Estado se incluye a } \\
\text { instituciones, bancos públicos y municipios. Para "otros" se } \\
\text { incluye a agencias de cooperación internacional y certificadoras }\end{array}$ \\
\hline Rol del Estado $(* *)$ & $\begin{array}{l}\text { Intermediario, comprador, } \\
\text { ambos y sin rol }\end{array}$ & Modalidad en la cual participa el Estado en el proyecto \\
\hline Tipo de transacción $\left(^{*}\right)$ & Dinero, en especie y mixto & Forma de pago a los proveedores del SE \\
\hline $\begin{array}{l}\text { Costo de oportunidad } \\
\qquad(* * *)\end{array}$ & No, no especificado, sí & $\begin{array}{l}\text { Inclusión del costo de oportunidad de los proveedores como } \\
\text { determinante del valor (monetario o en especie) a retribuirle }\end{array}$ \\
\hline $\begin{array}{l}\text { Tenencia de la tierra } \\
\qquad(* * *)\end{array}$ & $\begin{array}{l}\text { Privado, público, } \\
\text { comunitario, mixta }\end{array}$ & $\begin{array}{c}\text { Forma legal de tenencia de la tierra de los proveedores. Se } \\
\text { indicó además la existencia de tenencia precaria de la tierra } \\
\text { (posesión inestable generalmente asociada a carencia de título } \\
\text { de propiedad). La clase "mixta" incluye situaciones en las que } \\
\text { está presente más de una forma de tenencia }\end{array}$ \\
\hline $\begin{array}{l}\text { Objetivo relacionado } \\
\text { con pobreza }(* * *)\end{array}$ & No, no aplica y sí & $\begin{array}{l}\text { Se indicó si el proyecto incluía objetivos explícitos relacionados } \\
\text { con la pobreza, desarrollo rural y/o calidad de vida }\end{array}$ \\
\hline Condicionalidad $(* * *)$ & No, no especificado y sí & $\begin{array}{l}\text { La condicionalidad refiere a que la compensación al proveedor } \\
\text { se realiza únicamente en caso de que haya evidencias de que } \\
\text { haya cumplido con lo establecido en los objetivos del proyecto }\end{array}$ \\
\hline Efectividad $(* *)$ & $\begin{array}{l}\text { Efectivo, no efectivo y no } \\
\text { especificado }\end{array}$ & $\begin{array}{l}\text { Se definió la efectividad a partir de lo declarado en las } \\
\text { publicaciones respecto al nivel de cumplimiento de los objetivos } \\
\text { ambientales de cada proyecto. Los objetivos generalmente } \\
\text { están asociados con el cambio en la cantidad y/o calidad del SE } \\
\text { provisto }\end{array}$ \\
\hline Adicionalidad $(* * *)$ & No, no especificado, sí & $\begin{array}{l}\text { El nivel de adicionalidad fue caracterizado para aquellos } \\
\text { proyectos que se determinaron como efectivos. Un proyecto es } \\
\text { adicional cuando de no existir la compensación no se hubiese } \\
\text { asegurado la provisión del servicio (Tacconi 2013) }\end{array}$ \\
\hline $\begin{array}{l}\text { Equidad en cuanto } \\
\text { al Acceso (A), en la } \\
\text { Inclusión en la toma } \\
\text { de decisiones (I) y } \\
\text { en la Distribución de } \\
\text { beneficios y costos (D) } \\
\qquad(* * *)\end{array}$ & $\begin{array}{l}\text { Equitativo, no aplica, no } \\
\text { equitativo, no especificado }\end{array}$ & $\begin{array}{l}\text { El nivel de equidad fue representado con las clases "Equitativo" } \\
\text { y "No equitativo". Se asignaron cuando las referencias indicaron } \\
\text { de forma consistente (i.e., mediante la presentación de datos o } \\
\text { citas bibliográficas) el grado de incorporación y cumplimiento } \\
\text { de los elementos que las conforman. En los proyectos que no } \\
\text { documentaron el tratamiento de la dimensión de equidad en } \\
\text { cuestión, y cuando no estuvieron involucrados actores sociales } \\
\text { con niveles socioeconómicos desfavorables, se asignaron las } \\
\text { clases "no especificado" y "no aplica", respectivamente }\end{array}$ \\
\hline Metodología (**) & $\begin{array}{l}\text { Cualitativa, cuantitativa, } \\
\text { ambas, no especificado }\end{array}$ & $\begin{array}{l}\text { Se detalla la metodología utilizada en las publicaciones para } \\
\text { determinar la efectividad y la equidad de los proyectos }\end{array}$ \\
\hline
\end{tabular}


de los actores en las actividades y en la toma de decisiones, $\mathrm{b}$ ) reconocimiento, que implica tener en cuenta las normas, valores $\mathrm{y}$ formas de conocimiento que poseen los actores, c) distribución, que hace referencia a la distribución de costos y beneficios, y d) contexto, que hace alusión a las condiciones preexistentes que determinan la capacidad e influencia que tienen los actores en la toma de decisiones. Calvet-Mir et al. (2015) modificaron levemente estas dimensiones para caracterizarlas de forma más operativa. Las dimensiones que describen, y en este trabajo se utilizan, son la equidad en el acceso (acceso de los actores sociales a las actividades del proyecto), en la inclusión (i.e., procedimiento sensu McDermott et al. 2013) y en la distribución (mismo nombre sensu McDermott et al. 2013). En este trabajo se evaluaron las diferentes dimensiones de la equidad social para los proveedores de los SE (es decir, aquellos actores que reciben o potencialmente podrían recibir una compensación).

\section{Análisis de datos}

Para el análisis cuantitativo de la asociación de la efectividad y la equidad entre sí, y con el resto de las características analizadas, se realizaron pruebas exactas de Fisher (Agresti and Kateri 2011). Este método es el más apropiado para analizar la relación entre variables categóricas cuando el tamaño de muestra es pequeño (McDonald 2009; Agresti and Kateri 2011). El análisis se realizó con el software R.

\section{Resultados}

Las fuentes de información que reportaron los proyectos analizados fueron igualmente repartidas entre informes y artículos científicos, con el $42 \%$ cada uno, mientras que el $16 \%$ restante fueron mixtas (Figura 1). Con respecto a la escala espacial, $68 \%$ de los casos ocurre a escala local, $22 \%$ a escala regional y $10 \%$ a escala nacional. Un $77 \%$ de los proyectos son de corto plazo y un $23 \%$ de plazo medio, en tanto que no se registraron proyectos de largo plazo. Los SE provistos estuvieron en un $60 \%$ de los casos vinculados a la dinámica y a la provisión de agua, en un $13 \%$ al mantenimiento de la biodiversidad, en un $10 \%$ a la dinámica del carbono y en un $17 \%$ de los casos consideraron múltiples SE
(Figura 1). De los 60 programas analizados, 10 proceden de Bolivia, 9 de Costa Rica, 8 de Brasil, Colombia y Ecuador, 7 de México, 4 de Nicaragua y 2 de El Salvador. Por su parte, países como Belice, Guatemala, Honduras y Perú presentan un programa cada uno (Figura $1)$.

Los proveedores del servicio son privados en el $86 \%$ de los casos; públicos, en el $4 \%$ y el $10 \%$ restante son mixtos. Mientras tanto, los usuarios son $37 \%$ privados, $28 \%$ públicos y $35 \%$ mixtos (Figura 1). El 57\% de los usuarios son nacionales, el $26 \%$ son nternacionales y el $27 \%$ son de ambos orígenes. Sólo en 4 casos (7\%) no existieron intermediarios entre usuarios y proveedores. De los 56 casos restantes, entre los intermediarios estuvieron presentes el Estado (77\% de los proyectos), ONG $(62 \%)$ y otros (e.g., agencias de cooperación internacional y certificadoras) (13\%). El Estado ofició como intermediario en el 33\% de los programas, como usuario en el $7 \%$, como usuario e intermediario simultáneamente en el $44 \%$ y no tuvo un rol específico en el $16 \%$ restante (Figura 1).

La tenencia de la tierra en los territorios donde se llevaron a cabo los proyectos fue, en la mayoría de los casos, privada (38\%), seguida por mixta $(35 \%)$, pública $(8 \%)$ y comunitaria (5\%), mientras que no estuvo especificada en el 14\% de los casos (Figura 1). En $30 \%$ de los proyectos, la tenencia de la tierra fue precaria, en tanto que en el 50\% existieron formas estables de tenencia y en $20 \%$ no se especificó esta situación. Los tipos de transacción registrados entre compradores y vendedores fueron en dinero $(33 \%$ de los casos), en especies (19\%) y ambos (48\%). En $55 \%$ de los proyectos no estuvieron presentes objetivos explícitos relacionados con pobreza, desarrollo rural y/o calidad de vida de la población local, y fueron incluidos en $43 \%$ (Figura 1).

Los proyectos resultaron efectivos en cuanto a sus objetivos ambientales en el $43 \%$ de los casos, mientras que en el $20 \%$ resultaron no efectivos y en el $37 \%$ restante la efectividad no fue especificada (Figura 2). Sólo $8 \%$ de los proyectos presentaron adicionalidad. La condicionalidad fue incluida como requisito en $39 \%$ de los proyectos, mientras que en la mitad de los proyectos no fue especificada (Figura 2). En aproximadamente la mitad de los proyectos, la compensación al proveedor del servicio se basó en su costo de oportunidad (Figura 2). 


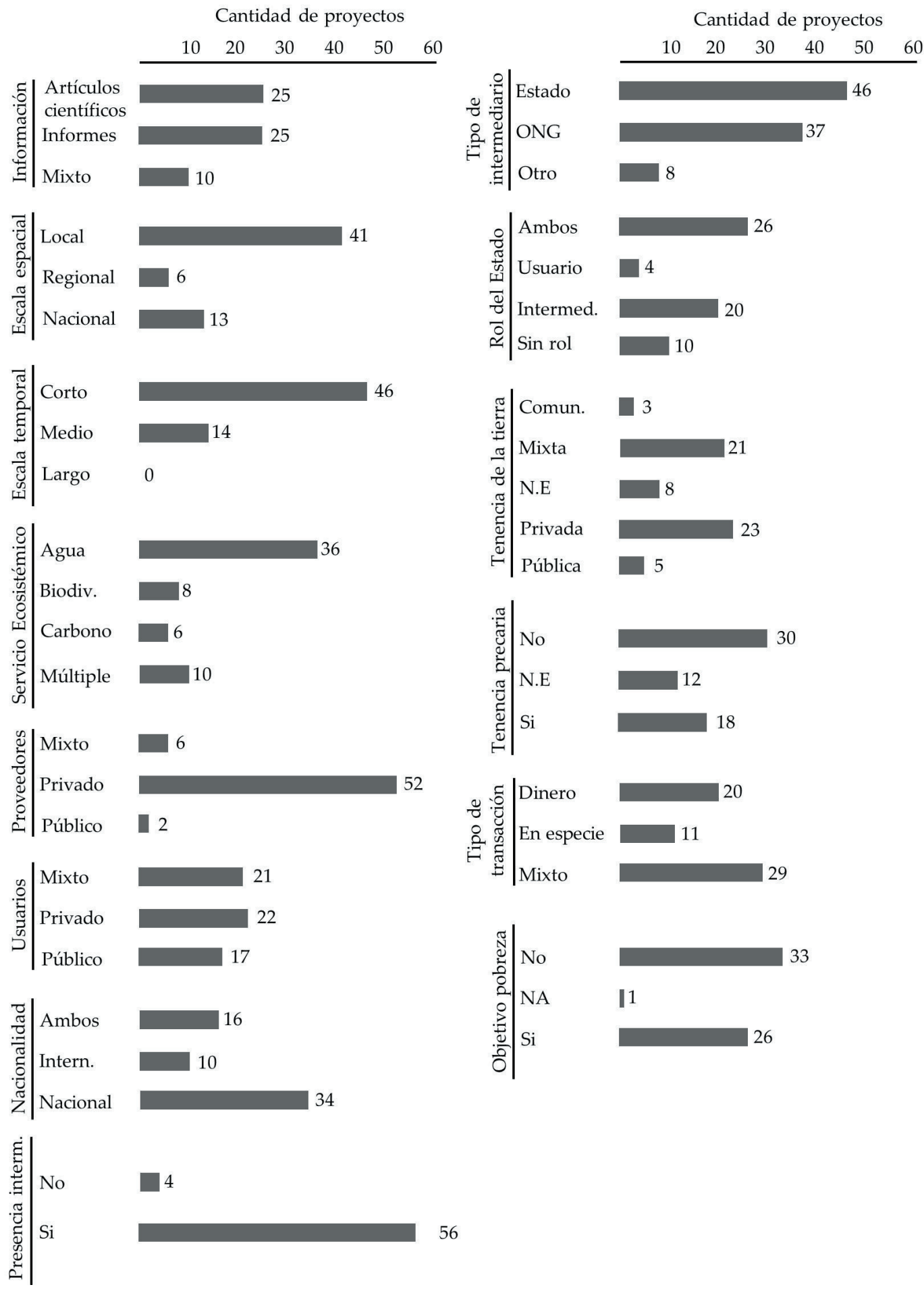

Figura 1. Número de proyectos que incluyen instrumentos económicos para la conservación de la naturaleza (total 60) según las categorías identificadas en la Tabla 1. Se muestran los resultados referidos a características generales y socioeconómicas de los proyectos y de las partes involucradas en la transacción. Biodiv., Intern., Interm., Comun. y N.E. significan biodiversidad, internacional, intermediario, comunitaria y no especificado, respectivamente.

Figure 1. Number of projects that include economic instruments for nature conservation (total 60) grouped by the categories identified in the Table 1 . The results referred to the general and socio-economic characteristics of the projects and of the agents involved are plotted. 


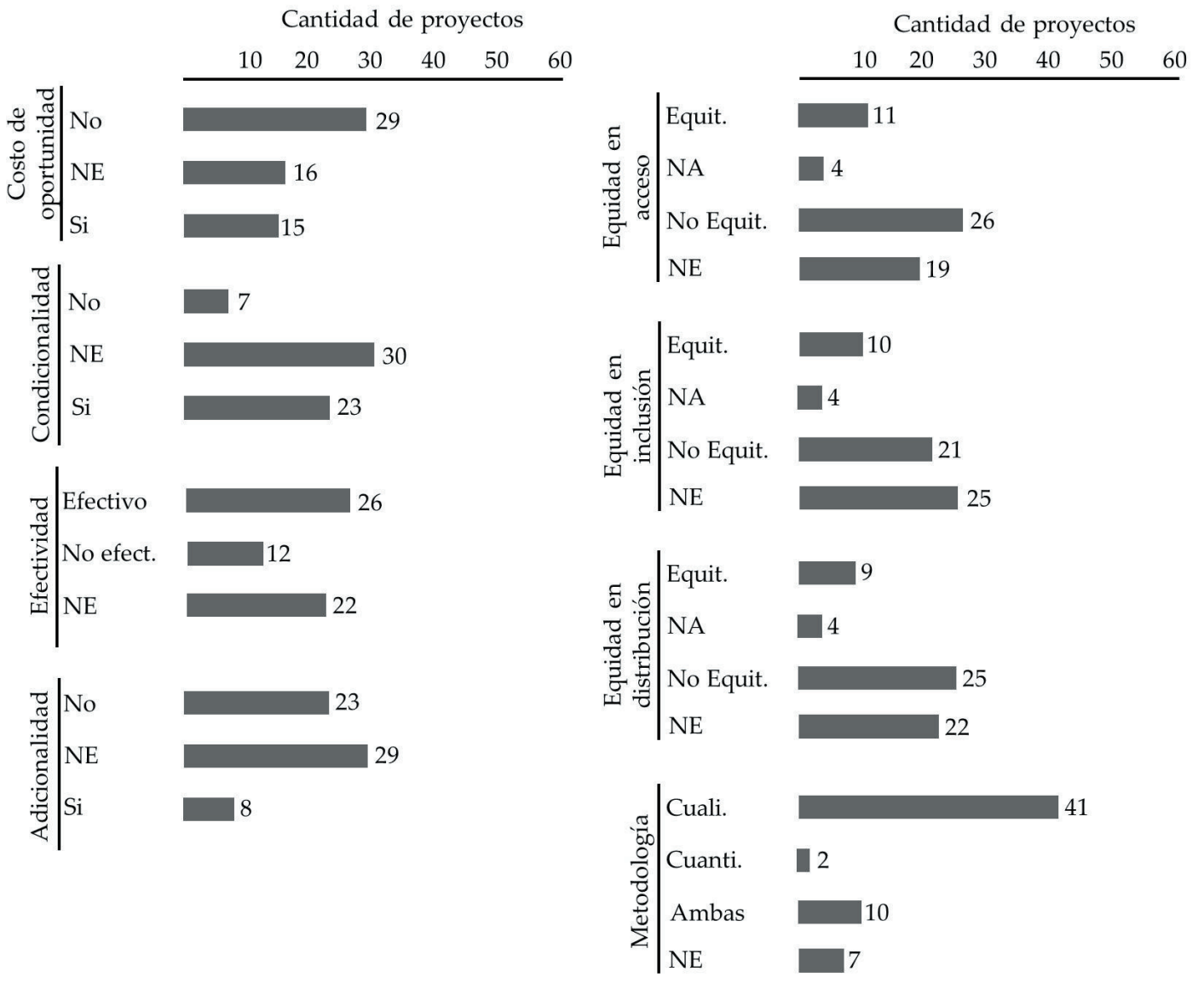

Figura 2. Número de proyectos que incluyen instrumentos económicos para la conservación de la naturaleza (total 60) según las categorías identificadas en la Tabla 1 . Se muestran los resultados referidos a la efectividad y equidad. NE, NA, Cuali., Cuanti., significan No especificado, No aplica, Cualitativo y Cuantitativo, respectivamente.

Figure 2. Number of projects that include economic instruments for nature conservation (total 60) grouped by the categories identified in the Table 1 . Results referred to the effectiveness and social equity are plotted.

Con respecto al análisis de las dimensiones de equidad social, el $43 \%$ resultó no equitativa en la dimensión de acceso, mientras que equitativa y no especificada en el 18 y $32 \%$ de los proyectos, respectivamente (Figura 2). Para la dimensión de la inclusión en la toma de decisiones, el 35\% de los casos fueron evaluados como no equitativos, $17 \%$ fueron equitativos y no especificada en $42 \%$ de los proyectos. La equidad en la distribución de beneficios y costos fue no equitativa en el $42 \%$ de los proyectos mientras que no especificada en el 37\% y equitativa en el 15\% (Figura 2). Para todas las dimensiones de equidad hubo cuatro casos en los que no correspondió describirla porque hubo un solo actor (el proveedor del servicio).

Tanto la efectividad como la equidad suelen ser independientes de las distintas características evaluadas (Tabla 2), pues únicamente el 20\% de las asociaciones evaluadas resultaron significativas. La relación entre todas las variables tampoco mostró gran cantidad de asociaciones significativas (Tabla 2 del material suplementario). La efectividad tiende a estar más especificada cuando el Estado interviene con doble rol (i.e., comprador e intermediario); en estos casos, la efectividad es a menudo mayor que cuando sólo participa de una de las dos formas (Figura 3.A). Por lo general, los proyectos orientados a la provisión de agua tienen especificada en menor proporción la adicionalidad respecto al resto de los SEs, y en los casos en que sí está especificada, no hay evidencias de adicionalidad (Figura 3B). A su vez, los informes suelen especificar, en menor medida, la adicionalidad respecto de las publicaciones científicas, y estas últimas tienden a determinar, en mayor medida, que no existe adicionalidad respecto a los informes (Figura 3C).

Las cuatro dimensiones de la equidad están asociadas significativamente (Tabla 2). Esto 
Tabla 2. Asociación entre la efectividad y las dimensiones de la equidad social con variables sociales, económicas e institucionales de los proyectos. La Tabla 1 contiene la descripción de las variables. Los valores de $P$ provienen de la prueba exacta de Fisher. Únicamente figuran aquellas asociaciones que resultaron significativas $(P<0.05)$. En la información suplementaria están las asociaciones entre todas las variables.

Table 2. Association between effectiveness and social equity with social, economic and institutional variables of the projects. Table 1 contains the description of the variables. The p-values were obtained from Fisher exact test. The $P$ values were obtained from Fisher exact test. Only significant associations are shown $(P<0.05)$.

\begin{tabular}{|c|c|c|c|c|c|}
\hline & 19. Efectividad & 20. Adicionalidad & $\begin{array}{l}\text { 21. Equidad } \\
\text { acceso }\end{array}$ & $\begin{array}{l}\text { 22. Equidad } \\
\text { decisiones }\end{array}$ & $\begin{array}{l}\text { 23. Equidad } \\
\text { distribución }\end{array}$ \\
\hline 1. Procedencia información & & 0.016 & 0.032 & 0.037 & \\
\hline 2. Escala espacial & & & & & \\
\hline 3. Escala temporal & 0.021 & & & & \\
\hline 4. Servicio ecosistémico & & 0.03 & & & \\
\hline 5. Proveedores & & & & & \\
\hline 6. Usuarios & & & & & \\
\hline 7. Nacionalidad usuario & & & & & \\
\hline 8. Estado & & & 0.025 & & 0.037 \\
\hline 9. ONG & & & & & \\
\hline 10. Otros & & & & & \\
\hline 11. Rol del Estado & 0.02 & & & & \\
\hline 12. Transacción & & & 0.024 & & \\
\hline 13. Condicionalidad & & & 0.014 & & \\
\hline 14. Costo de oportunidad & & & & & \\
\hline 15. Tenencia & & & & & \\
\hline 16. Tenencia precaria & & & & & \\
\hline 17. Objetivo pobreza & & & 0.002 & 0.004 & 0.002 \\
\hline 18. Metodología & & & & & \\
\hline 19. Efectividad & & 0 & & & \\
\hline 20.Adicionalidad & 0 & & & & \\
\hline 21. Equidad acceso & & & & 0 & 0 \\
\hline 22. Equidad decisiones & & & 0 & & 0 \\
\hline 23. Equidad distribución & & & 0 & 0 & \\
\hline
\end{tabular}

implica que comúnmente, cuando una de las dimensiones es considerada de una forma (e.g. equitativa o no equitativa), las demás dimensiones también son consideradas igual,. A su vez, cuando una se asocia de forma positiva con otra de las variables, las demás dimensiones también se comportan así. Los artículos científicos suelen reportar, en mayor medida, la equidad en el acceso respecto a los informes, y también suelen determinar que ésta no resultó efectiva (Figura 3D). Cuando el proyecto incluye la presencia del Estado como intermediario, es frecuente que la equidad en cuanto al acceso esté menos especificada en relación a cuando el Estado está ausente en este rol (Figura 3E). Además, cuando sí está especificada la equidad en el acceso no hay evidencia de que haya diferencias entre la presencia o no del Estado como intermediario en cuanto a la equidad en esta dimensión. Si la transacción se realiza en dinero, la equidad en el acceso suele ser menor respecto a cuando se realiza en especie (Figura 3F). La equidad en el acceso suele ser menos equitativa cuando hay condicionalidad (Figura 3G). Por último, la inclusión de objetivos específicos relacionados con la pobreza no implica que los proyectos sean más equitativos en el acceso, pero sí que se especifique menos si lo fueron (Figura 3H).

\section{Discusión}

\section{Efectividad y equidad de los IEBMC en LA}

Nuestros resultados indican que un poco menos de la mitad de los proyectos incluidos en esta revisión fueron efectivos, mientras que una cantidad levemente menor no tiene especificada su efectividad. De los efectivos, la mitad no cuenta con una evaluación respecto a su adicionalidad, es decir, acerca de si los beneficios asociados a un cambio de manejo, de uso de la tierra o de la provisión de un servicio hubiesen ocurrido sin la presencia del proyecto. De los que sí la incluyen, únicamente ocho proyectos presentaron adicionalidad. Esto sugiere que no hay 

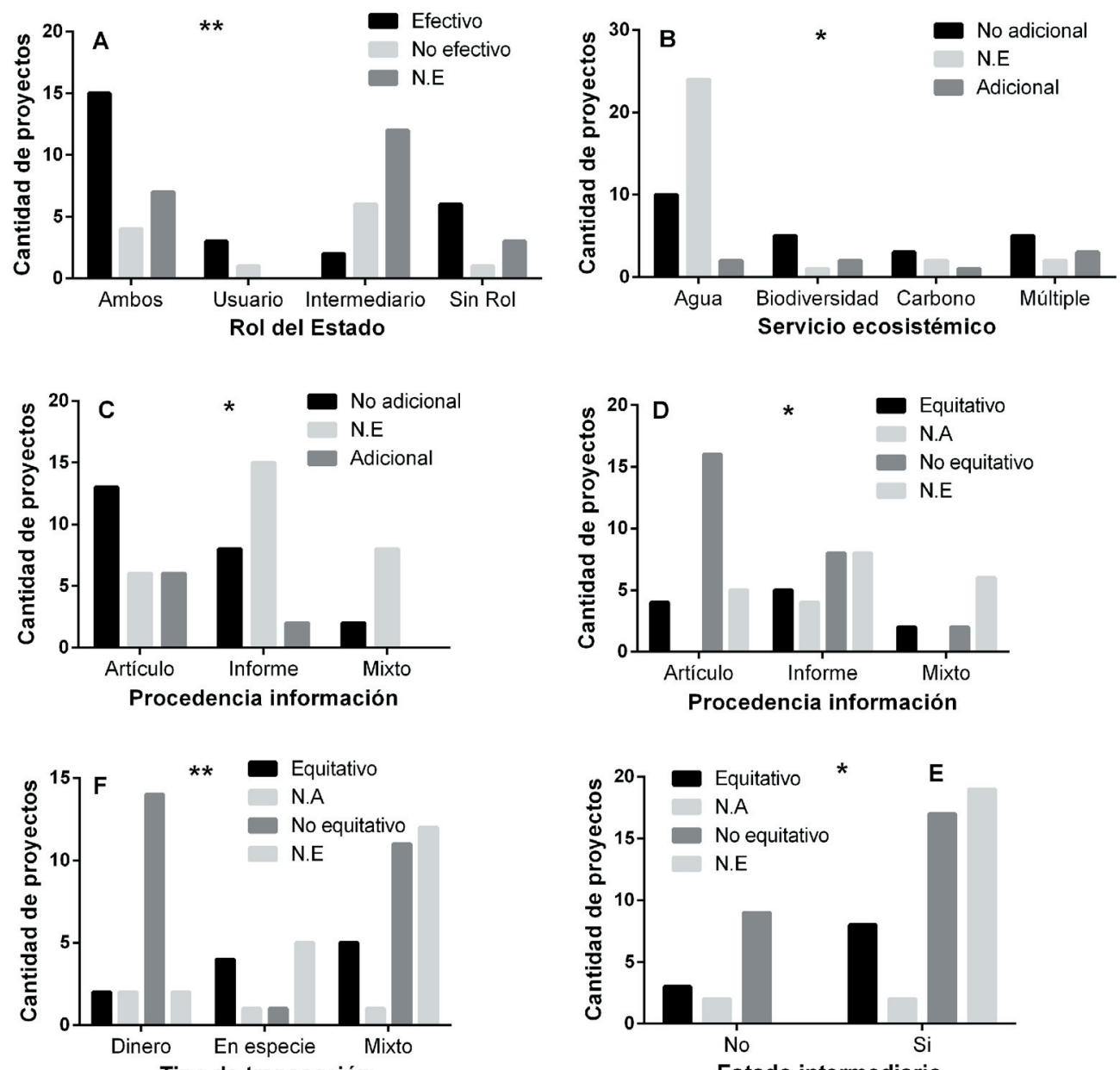

Tipo de transacción
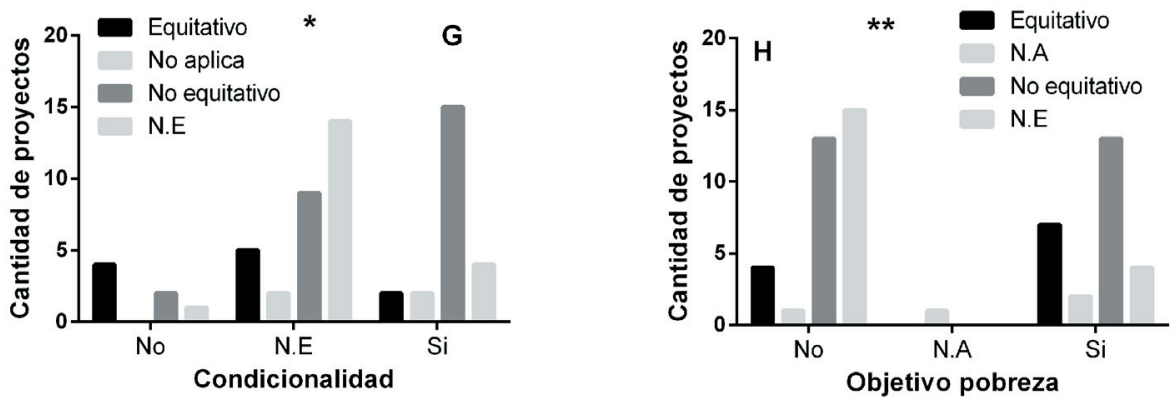

Figura 3. Relación entre la efectividad y equidad social de los proyectos y diferentes características de estos. Los asteriscos indican el nivel de significancia estadística $\left({ }^{*}: \mathrm{P}<0.05 ;{ }^{* *}: \mathrm{P}<0.01 ;{ }^{* * *}: \mathrm{P}<0.001\right)$; n.s. indica no significativo. La significancia estadística proviene de la prueba exacta de Fisher. N.E: No especificado. A: Relación entre el rol del Estado y la efectividad de los proyectos. B: Relación entre el servicio ecosistémico transado y la adicionalidad. C: Relación entre la procedencia de la información y la adicionalidad. D: Relación entre procedencia de la información y equidad en el acceso. E: Relación entre presencia del Estado como intermediario y equidad en el acceso. F: Relación entre el tipo de transacción y la equidad en el acceso. G: Relación entre condicionalidad y equidad en el acceso. H: Relación entre objetivo explícito relacionado con pobreza y equidad en el acceso.

Figure 3. Relation between the effectiveness and social equity of the analyzed projects and different characteristics of these. Asterisks indicate the level of statistical significance ( $\left.{ }^{*}: \mathrm{P}<0.05 ;{ }^{* *}: \mathrm{P}<0.01 ;{ }^{* * *}: \mathrm{P}<0.001\right) ;$ n.s. indicates not significant. Statistical significance comes from Fisher exact test. N.E: non-specified. A: Relationship between the role of the State and the effectiveness of the projects. B: Relationship between the ecosystem service and additionality. C: Relationship between the origin of the information and additionality. D: Relationship between the origin of information and equity in access. E: Relationship between the presence of the State as an intermediary and equity in access. F: Relationship between the type of transaction and equity in access. G: Relationship between conditionality and equity in access. H: Relationship between the explicit inclusion of a poverty related objective and equity in access. 
evidencias fuertes para considerar que la aplicación de IEBMC en LA haya sido exitosa en cuanto a los objetivos ambientales que se proponen. La condicionalidad (i.e., realizar la compensación al proveedor únicamente si hay evidencias de la prestación del servicio) es una de las características distintivas de los PES pues garantiza su provisión (Wunder 2015). Nuestros resultados sugieren que en la mitad de los casos no fue especificada y que aproximadamente en la mitad restante sí fue incluida como requisito para recibir la compensación. A su vez, la tenencia de la tierra resultó precaria en 30\% de los proyectos y no especificada en $20 \%$. Esto implica que una gran proporción de los proyectos evaluados no satisfacen los requisitos propuestos por Wunder (2005), por lo cual nuestros resultados coinciden con los de MartínOrtega et al. (2010) quienes concluyen que hay una gran divergencia entre la propuesta teórica de algunos de estos instrumentos y su aplicación real.

La mayoría de los proyectos no fueron equitativos en las diferentes dimensiones evaluadas. Esto es congruente con lo encontrado por Calvet-Mir et al. (2015) para un conjunto de proyectos ejecutados en países tropicales. En general, los proyectos que son equitativos en alguna de las dimensiones tienden a serlo también en las demás. Sin embargo, otros autores han encontrado casos en los que hubo equidad en la distribución de los ingresos, pero no en la inclusión de actores sin títulos de propiedad (Corbera et al. 2007). A su vez, nuestros resultados sugieren que existe menos equidad en el acceso cuando los proyectos incluyen cláusulas de condicionalidad. Esto sugeriría que la condicionalidad es un requisito que limita el acceso a la compensación; es decir: no acceden a la compensación ciertos actores sociales que a priori no reúnen características para ser potenciales proveedores. La inclusión de objetivos explícitos relacionados con la pobreza no implica que los proyectos sean más equitativos en el acceso, lo cual indica que la inclusión de estos objetivos no garantiza que se cumplan.

Los proyectos analizados fueron en mayor proporción efectivos que equitativos (e.g., $43 \%$ efectivos vs. $18 \%$ equitativos en acceso), más allá de la dimensión considerada de esta última. Por su parte, la equidad y la efectividad generalmentenoestán asociadas, loqueimplica que no hay sinergias ni compromisos entre ellas, sino que podrían ser independientes. En proyectos ejecutados en países tropicales se encontraron resultados similares (CalvetMir et al. 2015): los proyectos efectivos tienen tanto efectos sociales equitativos como inequitativos. Usualmente, cuando estos instrumentos son propuestos desde el Estado, tiende a haber mayor inclusión de objetivos sociales (Wunder 2013). No obstante, nuestros resultados sugieren que la equidad en el acceso no está garantizada cuando el Estado es el promotor del instrumento. Esto podría deberse a la exclusión de aquellos actores sociales que carecen de títulos de propiedad, lo que, en algunos proyectos, resulta una restricción para acceder a la compensación. Esto sugiere que los IEBMC no son en sí una forma sinérgica de conservar la naturaleza y aliviar la pobreza rural (Muradian et al. 2013; Pinho et al. 2014); por el contrario, la relación entre ambas es compleja y requiere abordajes acordes a ello (Daw et al. 2011). Sin embargo, como indican Pascual et al. (2014), las sinergias entre la efectividad y la equidad pueden ocurrir en plazos superiores a los del proyecto particular. En definitiva, la inclusión de objetivos sociales en los IEBMC está comenzando a tomar relevancia en el diseño de estos instrumentos (Pascual et al. 2014), aún cuando algunos autores consideren que los objetivos de los proyectos no deberían necesariamente incluir una mejora en las condiciones sociales (Barrett et al. 2011; Wunder 2013).

La descripción de las diferentes características de los proyectos y su relación con la efectividad y equidad muestran que los IEBMC presentes en LA son diversos en cuanto al tipo de actores involucrados, a sus relaciones y a cómo éstas determinan los resultados de los proyectos. Esta diversidad es un reflejo de la complejidad socio-ecológica en la cual se insertan los proyectos y que hace necesaria su adaptación a diferentes sistemas de gobernanza (Engel et al. 2008; Vatn 2010). La escasa o nula asociación estadística de la efectividad y la equidad con el resto de las características sugiere que no existe un arreglo institucional único que asegure el buen desempeño de estos instrumentos. A su vez, la baja asociación o independencia entre estas variables podría indicar que el contexto en el que se implementan los instrumentos tiene poca incidencia en su desempeño. Esto implicaría que el diseño (i.e., objetivos, acciones) de los diferentes proyectos no fue apropiado para resolver los problemas socio-ecológicos que tenían como objetivo solucionar. Sin embargo, otros estudios comparativos sugieren que existen ciertos atributos que si afectan el 
desempeño. Por ejemplo, los programas son más efectivos cuando se los diseña de forma colaborativa entre compradores y vendedores, y menos efectivos cuando el gobierno actúa como comprador (Wunder et al. 2008). En definitiva, como sucede con cualquier otra propuesta de manejo de los recursos naturales, no existen panaceas en el diseño de políticas ambientales para maximizar su efectividad y equidad social. Por el contrario, diferentes condiciones socio-ecológicas hacen necesario que los proyectos se adapten al contexto cultural e histórico local donde se aplican y generen arreglos institucionales acordes a estas condiciones (Ostrom 2007; Corbera et al. 2009). Se requiere más evidencia empírica para determinar en qué medida el desempeño de los IEBMC es explicado por el instrumento o por su contexto de aplicación.

Las aproximaciones utilizadas para caracterizar la efectividad fueron múltiples ; van desde evaluaciones biofísicas de la provisión del SE o del uso de la tierra hasta percepciones de los actores involucrados en la transacción. A su vez, la caracterización de las diferentes dimensiones de la equidad por lo general se basa sobre una apreciación subjetiva. Esto coincide con Calvet-Mir et al. (2015), quienes remarcan que las evaluaciones cuantitativas son muy escasas. La diversidad de formas de evaluar la efectividad dificulta la comparación a través de proyectos, lo cual es una limitación de este trabajo. Por ello, al igual que en estudios recientes (Prager et al. 2015), encontramos deficiencias graves en la calidad de la información referida al monitoreo y la evaluación de los proyectos, lo que podría deberse, entre otras razones, tanto a la ausencia de estas actividades como al carácter privado de la información generada. Esto, junto con la ausencia frecuente de situaciones de control, limita seriamente el alcance de las experiencias presentadas y la capacidad de realizar inferencias acerca de la contribución del diseño y la implementación al desempeño de los proyectos. Creemos que incorporar el marco del co-manejo adaptativo (Kofinas 2009) podría ser una contribución clave para mejorar social y ambientalmente estos instrumentos. Esto requeriría, por un lado, que los proyectos incluyan en su diseño e implementación actividades de monitoreo y evaluación (Naeem et al. 2015) para que de forma iterativa los actores involucrados aprendan del proceso identificando sus debilidades y puedan adaptarse a condiciones socio-ecológicas dinámicas en contextos de incertidumbre (Kofinas 2009). El aspecto iterativo es crítico en este tipo de proyectos ya que su dinámica generalmente modifica el papel de los actores, las formas de pago, etc. (Raes et al. 2016). La inclusión del componente de "co" en el manejo de los proyectos, hace referencia a la necesidad de incluir a los diferentes actores sociales involucrados en la toma de decisiones, pues esto no solo podría afectar la equidad y legitimidad del proyecto, sino también contribuir a su efectividad (Ribot and Larson 2012; Kinzig et al. 2013). En tal sentido, los resultados obtenidos en este trabajo permiten analizar el diseño e implementación de los esquemas y no a los IEBMC como una herramienta de gestión ambiental. Es decir, estos instrumentos no deberían ser necesariamente excluidos de la gobernanza ambiental por carecer de un diseño e implementación adecuados.

\section{Los IEBMC y el principio precautorio}

Los resultados presentados abordan una de las dimensiones desde la que pueden ser evaluados los IEBMC: su efectividad. Los análisis sugieren que no hay evidencias fuertes de sus ventajas en cualquier circunstancia. Más aun, los problemas de diseño y documentación plantean serios problemas para la evaluación de su efectividad. Sin embargo, los IEBMC pueden (y deben) ser analizados y criticados desde otros planos, ortogonales al de su efectividad, como el ético, el político y el ideológico. Desde esa perspectiva hay dos dimensiones a considerar. Por un lado, estos mecanismos asignan a la naturaleza un valor de cambio, la mercantilizan (Kosoy and Corbera 2010; Silvertown 2015). Por otro, introducir estos instrumentos tiene efectos en las partes que participan de la transacción. Respecto a la primera cuestión, los aportes monetarios pueden ser una forma de cambiar la percepción de la naturaleza que tienen ciertas culturas, al pasar de una postura de valoración intrínseca a una de tipo instrumental. Esto puede ser perjudicial para sociedades en las que la lógica utilitarista está ausente y la participación en mercados es reducida (e.g., muchas comunidades rurales de LA) (Gómez-Baggethun et al. 2010). Respecto a los efectos sobre las partes que participan de la transacción, Sandel (2012) y Satz (2010) señalan que los mercados pueden no ser neutros en cuanto a las consecuencias que tienen sobre quienes participan en las transacciones. Satz (2010) habla de "mercados nocivos" cuando la transacción ocurre en 
condiciones de distribución asimétrica de información o poder. Algunas de las consecuencias de implementar "mercados nocivos" pueden analizarse en torno a las diferentes dimensiones de la equidad como hemos hecho en este artículo (McDermott et al. 2013; Pascual et al. 2014). Sin embargo, una de las dimensiones no consideradas por la dificultad de su evaluación es la equidad de contexto. En este sentido, la gran mayoría de los IEBMC muestran una enorme asimetría entre compradores (e.g., ONG, Estados, grandes compañías) y proveedores (e.g., comunidades campesinas, pueblos originarios) respecto de recursos, información y posibilidades de participación, lo cual podría comprometer seriamente el carácter voluntario de estos mecanismos. En este sentido, Ribot and Larson (2012) remarcan que aunque muchos proyectos incluyan procesos participativos, esta participación puede significar cosas muy diferentes y puede llevar en algunos casos a un consenso coercitivo debido a la disparidad de poder y acceso a la información.

En el plano internacional, estas asimetrías se pueden reflejar en las relaciones centroperiferia(Cardosoand Faletto 1996; Wallerstein 2004), en las que los IEBMC podrían ser una forma más de mantener estas dinámicas, y retroalimentar la denominada "maldición de los recursos naturales" (Kronenberg and Hubacek 2013). En este contexto, existen ejemplos de acaparamientos de tierras en LA con fines turísticos (i.e., SE culturales) y de captura de carbono (Anseeuw et al. 2013; Costantino 2014) que muestran cómo pueden llegar a operar estas asimetrías. A este proceso de acaparamiento de tierras y/o de recursos naturales con fines ambientales, algunos autores lo han llamado acaparamiento verde (del inglés "green grabbing") (Fairhead et al. 2012). En este contexto, resulta crítico discutir cómo este proceso puede afectar el desarrollo de los países periféricos y cuál sería su efecto sobre las condiciones de vida de la población local.

La falta parcial de evidencia y certeza respecto a los resultados ambientales, económicos y sociales de los IEBMC en LA debería alertar a quienes toman decisiones. En este contexto, la gobernanza ambiental en LA se encuentra frente a una encrucijada. En esta región, la reducida efectividad de las medidas implementadas por los gobiernos y los riesgos sociales de los IEBMC plantean un dilema. Este dilema se complejiza aún más debido a la elevada diversidad biológica y cultural de LA
(Balvanera et al. 2012), a la gran proporción de población con pobreza rural crónica (Vakis et al. 2015) y a una fuerte presión global para aprovechar sus recursos naturales (Lambin et al. 2013). En estas condiciones, los tomadores de decisiones podrían promover IEBMC de forma urgente a fin de garantizar la conservación de la naturaleza, independientemente de sus posibles efectos negativos, apelando así al principio precautorio. Este principio indica que frente a la amenaza de un daño ambiental se deben tomar acciones para controlar o disminuir tal riesgo, incluso cuando existe incertidumbre científica respecto a los efectos de dichas acciones (Kriebel et al. 2001). A diferencia de cómo se suele utilizar este principio, en este caso, los riesgos de los IEBMC no son ambientales sino éticos, sociales y políticos. Pensamos que apelar a este principio para promover los IEBMC sin profundizar la descripción de sus consecuencias sociales podría ser peligroso pues se obviarían otros aspectos esenciales de la sustentabilidad. Por ello, además de profundizar el conocimiento respecto a estos instrumentos, creemos necesario avanzar en nuevas herramientas de conservación que surjan de reconciliar las perspectivas de valoración intrínseca e instrumental (Loreau 2014), pensar en nuevas (Chan et al. 2016) o avanzar en alternativas no basadas en incentivos económicos y/o regulación gubernamental (Nyborg et al. 2016). Por ejemplo, la sesión de tierras a comunidades locales (Brondizio and Le Tourneau 2016), donde las motivaciones intrínsecas para la conservación son elevadas (ver Bowles et al. 2008) ha resultado ser una de las políticas más efectivas para reducir la deforestación en el Amazonas (Nolte et al. 2013).

\section{Consideraciones finales}

Los IEBMC y su aplicación han dado lugar a un interesante debate académico (Muradian et al. 2013; Wunder 2013) con visiones polarizadas (Tacconi 2012). Hasta la actualidad, este debate ha ocurrido principalmente en el plano teórico, aunque en los últimos tiempos, diferentes trabajos (Brouwer et al. 2011; Ezzine-de-Blas et al. 2016) han comenzado a resumir los proyectos vigentes para contribuir al debate con una aproximación empírica. Este trabajo busca ser un aporte en este sentido. Nuestros resultados indican que no hay evidencias fuertes para promover los IEBMC ya sea desde una perspectiva ambiental como social, y principalmente en LA, donde algunas de sus característicassocio-ecológicassuponenriesgos 
adicionales. Actualmente, las tendencias y el estado global de la biodiversidad y de gran cantidad de los SEs resultan alarmantes (MEA 2005; Ceballos et al. 2015). Sin embargo, no existe consenso respecto a cuáles son las mejores herramientas para frenar o revertir estas tendencias. El crecimiento de IEBMC para resolver estos problemas debería ser evaluado con más cautela, tanto por científicos como por tomadores de decisiones, para evitar aceptarlos como una panacea o como parte de un "pragmatismo ambiental" (Spash 2009). Para ello, se necesita una mayor y mejor articulación del sistema de ciencia y técnica con los tomadores de decisiones y con los actores sociales afectados, directa o indirectamente, por sus decisiones. En pos de eso, consideramos indispensable que el flujo de información entre los actores sociales involucrados vaya acompañado de una exposición explícita de sus valores e ideologías.

Agradecimientos. Agradecemos a Agostina Torres, Martín Aguiar y a dos revisores anónimos, cuyos comentarios contribuyeron a mejorar sustancialmente este artículo. El CONICET, la UBA, FONCYT y el Inter American Insitute (IAI) for Global Change Research, que es financiado por la US National Science Foundation (Grant GEO-1128040), a través del proyecto CRN3095 (Bridging Ecosystem Services and Territorial Planning (BEST-P): A southern South American intiative) financiaron la realización de este artículo.

\section{REFERENCIAS}

Agresti, A., and M. Kateri. 2011. Categorical data analysis. Springer.

Anseeuw, W., J. Lay, P. Messerli, M. Giger, and M. Taylor. 2013. Creating a public tool to assess and promote transparency in global land deals: the experience of the Land Matrix. Journal of Peasant Studies 40:521-530.

Balvanera, P., M. Uriarte, L. Almeida-Leñero, A. Altesor, F. DeClerck, T. Gardner, J. Hall, A. Lara, P. Laterra, and M. Peña-Claros. 2012. Ecosystem services research in Latin America: The state of the art. Ecosystem Services 2:56-70.

Barrett, C. B., A. J. Travis, and P. Dasgupta. 2011. On biodiversity conservation and poverty traps. Proceedings of the National Academy of Sciences 108:13907-13912.

Bowles, S. 2008. Policies designed for self-interested citizens may undermine" the moral sentiments": Evidence from economic experiments. Science 320:1605-1609.

Brouwer, R., A. Tesfaye, and P. Pauw. 2011. Meta-analysis of institutional-economic factors explaining the environmental performance of payments for watershed services. Environmental Conservation 38:380-392.

Bull, J. W., K. B. Suttle, A. Gordon, N. J. Singh, and E. Milner-Gulland. 2013. Biodiversity offsets in theory and practice. Oryx 47:369-380.

Calvet-Mir, L., E. Corbera, A. Martin, J. Fisher, and N. Gross-Camp. 2015. Payments for ecosystem services in the tropics: A closer look at effectiveness and equity. Current Opinion in Environmental Sustainability 14:150-162.

Cardoso, F. H., and E. Faletto. 1996. Dependencia y desarrollo en América Latina: ensayo de interpretación sociológica. Siglo XXI.

Ceballos, G., P. R. Ehrlich, A. D. Barnosky, A. García, R. M. Pringle, and T. M. Palmer. 2015. Accelerated modern human-induced species losses: Entering the sixth mass extinction. Science 1:e1400253.

Corbera, E., N. Kosoy, and M. M. Tuna. 2007. Equity implications of marketing ecosystem services in protected areas and rural communities: Case studies from Meso-America. Global Environmental Change 17:365-380.

Corbera, E., C. G. Soberanis, and K. Brown. 2009. Institutional dimensions of Payments for Ecosystem Services: An analysis of Mexico's carbon forestry programme. Ecological Economics 68:743-761.

Costantino, A. 2014. Land Grabbing in Latin America: Another Natural Resource Curse? Agrarian South: Journal of Political Economy 3:17-43.

Chan, K. M., P. Balvanera, K. Benessaiah, M. Chapman, S. Díaz, E. Gómez-Baggethun, R. Gould, N. Hannahs, K. Jax, and S. Klain. 2016. Opinion: Why protect nature? Rethinking values and the environment. Proceedings of the National Academy of Sciences 113:1462-1465.

Daw, T., K. Brown, S. Rosendo, and R. Pomeroy. 2011. Applying the ecosystem services concept to poverty alleviation: the need to disaggregate human well-being. Environmental Conservation 38:370-379.

Engel, S., S. Pagiola, and S. Wunder. 2008. Designing payments for environmental services in theory and practice: An overview of the issues. Ecological Economics 65:663-674.

Ezzine-de-Blas, D., S. Wunder, M. Ruiz-Pérez, and R. del Pilar Moreno-Sanchez. 2016. Global patterns in the implementation of payments for environmental services. PLoS One 11:e0149847.

Fairhead, J., M. Leach, and I. Scoones. 2012. Green Grabbing: a new appropriation of nature? Journal of Peasant Studies 39:237-261.

Galaz, V., J. Gars, F. Moberg, B. Nykvist, and C. Repinski. 2015. Why ecologists should care about financial markets. Trends in Ecology \& Evolution 30:571-580.

Gaston, K. J., S. F. Jackson, L. Cantú-Salazar, and G. Cruz-Piñón. 2008. The ecological performance of protected areas. Annual Review of Ecology, Evolution and Systematics 39:93-113. 
Gómez-Baggethun, E., R. De Groot, P. L. Lomas, and C. Montes. 2010. The history of ecosystem services in economic theory and practice: from early notions to markets and payment schemes. Ecological Economics 69:1209-1218.

Gómez-Baggethun, E., and R. Muradian. 2015. In markets we trust? Setting the boundaries of market-based instruments in ecosystem services governance. Ecological Economics 117:217-224.

Gómez-Baggethun, E., and M. Ruiz-Pérez. 2011. Economic valuation and the commodification of ecosystem services. Progress in Physical Geography 35:613-628.

Grima, N., S. J. Singh, B. Smetschka, and L. Ringhofer. 2016. Payment for Ecosystem Services (PES) in Latin America: Analysing the performance of 40 case studies. Ecosystem Services 17:24-32.

Gwynne, R. N., and C. Kay. 2014. Latin America transformed: globalization and modernity. Routledge.

Harvey, D. 2007. A brief history of neoliberalism. Oxford University Press, USA.

Ives, C. D., and S. A. Bekessy. 2015. The ethics of offsetting nature. Frontiers in Ecology and the Environment 13: 568-573.

Jenkins, M., S. J. Scherr, and M. Inbar. 2004. Markets for biodiversity services: potential roles and challenges. Environment: Science and Policy for Sustainable Development 46:32-42.

Kareiva, P., and M. Marvier. 2012. What is conservation science? BioScience 62:962-969.

Kinzig, A., C. Perrings, F. S. Chapin, S. Polasky, V. Smith, D. Tilman, and B. Turner. 2011. Paying for ecosystem servicespromise and peril. Science 334:603-604.

Kinzig, A. P., P. R. Ehrlich, L. J. Alston, K. Arrow, S. Barrett, T. G. Buchman, G. C. Daily, B. Levin, S. Levin, and M. Oppenheimer. 2013. Social norms and global environmental challenges: the complex interaction of behaviors, values, and policy. BioScience 63:164-175.

Kofinas, G. P. 2009. Adaptive co-management in social-ecological governance. Pp. 77-101 in Principles of ecosystem stewardship. Springer.

Kosoy, N., and E. Corbera. 2010. Payments for ecosystem services as commodity fetishism. Ecological Economics 69: 1228-1236.

Kriebel, D., J. Tickner, P. Epstein, J. Lemons, R. Levins, E. L. Loechler, M. Quinn, R. Rudel, T. Schettler, and M. Stoto. 2001. The precautionary principle in environmental science. Environmental Health Perspectives 109:871-76.

Kronenberg, J., and K. Hubacek. 2013. Could payments for ecosystem services create an "ecosystem service curse". Ecology and Society 18:10.

Lambin, E., H. Gibbs, L. Ferreira, R. Grau, P. Mayaux, P. Meyfroidt, D. Morton, T. Rudel, I. Gasparri, and J. Munger. 2013. Estimating the world's potentially available cropland using a bottom-up approach. Global Environmental Change 23:892-901.

Lockie, S. 2013. Market instruments, ecosystem services, and property rights: assumptions and conditions for sustained social and ecological benefits. Land Use Policy 31:90-98.

Loreau, M. 2014. Reconciling utilitarian and non-utilitarian approaches to biodiversity conservation. Ethics in Science and Environmental Politics 14:27-32.

Mace, G. M. 2014. Whose conservation? Science 345:1558-1560.

Martin-Ortega, J., E. Ojea, and C. Roux. 2013. Payments for water ecosystem services in Latin America: a literature review and conceptual model. Ecosystem Services 6:122-132.

Mascia, M. B., and S. Pailler. 2011. Protected area downgrading, downsizing, and degazettement (PADDD) and its conservation implications. Conservation Letters 4:9-20.

McDermott, M., S. Mahanty, and K. Schreckenberg. 2013. Examining equity: a multidimensional framework for assessing equity in payments for ecosystem services. Environmental Science \& Policy 33:416-427.

McDonald, J. H. 2009. Handbook of biological statistics. Sparky House Publishing Baltimore, MD.

Millenium Ecosystem Assessment (MEA). 2005. Ecosystems and human well-being. Island Press, Washington, D.C., USA.

Moreno-Sánchez, R. d. P. 2012. Incentivos económicos para la conservación: un marco conceptual. Iniciativa para la conservación en la Amazonía Andina (ICAA).

Muradian, R., E. Corber, U. Pascual, N. Kosoy, and P. H. May. 2010. Reconciling theory and practice: An alternative conceptual framework for understanding payments for environmental services. Ecological Economics 69(6):12021208.

Muradian, R., M. Arsel, L. Pellegrini, F. Adaman, B. Aguilar, B. Agarwal, E. Corbera, D. Ezzine de Blas, J. Farley, and G. Froger. 2013. Payments for ecosystem services and the fatal attraction of win-win solutions. Conservation Letters 6:274-279.

Naeem, S., J. Ingram, A. Varga, T. Agardy, P. Barten, G. Bennett, E. Bloomgarden, L. Bremer, P. Burkill, and M. Cattau. 2015. Get the science right when paying for nature's services. Science 347:1206-1207.

Norgaard, R. B. 2010. Ecosystem services: From eye-opening metaphor to complexity blinder. Ecological Economics 69:1219-1227.

Nyborg, K., J. M. Anderies, A. Dannenberg, T. Lindahl, C. Schill, M. Schlüter, W. N. Adger, K. J. Arrow, S. Barrett, and S. Carpenter. 2016. Social norms as solutions. Science 354:42-43.

Ostrom, E. 2007. A diagnostic approach for going beyond panaceas. Proceedings of the National Academy of Sciences 104:15181-15187. 
Pagiola, S., A. Arcenas, and G. Platais. 2005. Can payments for environmental services help reduce poverty? An exploration of the issues and the evidence to date from Latin America. World Development 33:237-253.

Pascual, U., R. Muradian, L. C. Rodríguez, and A. Duraiappah. 2010. Exploring the links between equity and efficiency in payments for environmental services: A conceptual approach. Ecological Economics 69:1237-1244.

Pascual, U., J. Phelps, E. Garmendia, K. Brown, E. Corbera, A. Martin, E. Gomez-Baggethun, and R. Muradian. 2014. Social equity matters in payments for ecosystem services. BioScience 64(11):1027-1036.

Pinho, P. F., G. Patenaude, J. P. Ometto, P. Meir, P. M. Toledo, A. Coelho, and C. E. F. Young. 2014. Ecosystem protection and poverty alleviation in the tropics: Perspective from a historical evolution of policy-making in the Brazilian Amazon. Ecosystem Services 8:97-109.

Pirard, R., and R. Lapeyre. 2014. Classifying market-based instruments for ecosystem services: A guide to the literature jungle. Ecosystem Services 9:106-114.

Prager, C., A. Varga, P. Olmsted, J. Ingram, M. Cattau, C. Freund, R. Wynn-Grant, and S. Naeem. 2015. An assessment of adherence to basic ecological principles by payments for ecosystem service projects. Conservation Biology.

Raes, L., L. Loft, J. F. Le Coq, G. Van Huylenbroeck, and P. van Damme. 2016. Towards market-or command-based governance? The evolution of payments for environmental service schemes in Andean and Mesoamerican countries. Ecosystem Services 18:20-32.

Ravallion, M. 2014. Income inequality in the developing world. Science 344:851-855.

Ribot, J., and A. Larson. 2012. Reducing REDD risks: affirmative policy on an uneven playing field. International Journal of the Commons 6.

Sandel, M. J. 2012. What money can't buy: the moral limits of markets. Macmillan.

Sattler, C., S. Trampnau, S. Schomers, C. Meyer, and B. Matzdorf. 2013. Multi-classification of payments for ecosystem services: how do classification characteristics relate to overall PES success? Ecosystem Services 6:31-45.

Satz, D. 2010. Why some things should not be for sale: The moral limits of markets. Oxford University Press.

Schomers, S., and B. Matzdorf. 2013. Payments for ecosystem services: A review and comparison of developing and industrialized countries. Ecosystem Services 6:16-30.

Schröter, M., E. H. Zanden, A. P. Oudenhoven, R. P. Remme, H. M. Serna-Chavez, R. S. Groot, and P. Opdam. 2014. Ecosystem services as a contested concept: a synthesis of critique and counter-arguments. Conservation Letters 7: 514-523.

Silvertown, J. 2015. Have ecosystem services been oversold? Trends in Ecology \& Evolution 30:641-648.

Spash, C. L. 2009. The new environmental pragmatists, pluralism and sustainability. Environmental Values 18:253256.

Tacconi, L. 2012. Redefining payments for environmental services. Ecological Economics 73:29-36.

Turner, W. R., K. Brandon, T. M. Brooks, C. Gascon, H. K. Gibbs, K. S. Lawrence, R. A. Mittermeier, and E. R. Selig. 2012. Global biodiversity conservation and the alleviation of poverty. BioScience 62:85-92.

Vakis, R., J. Rigolini, and L. Lucchetti. 2015. Left behind: chronic poverty in Latin America and the Caribbean. World Bank Publications.

Vatn, A. 2010. An institutional analysis of payments for environmental services. Ecological Economics 69:1245-1252.

Vatn, A. 2015. Markets in environmental governance. From theory to practice. Ecological Economics 117:225-233.

Wallerstein, I. M. 2004. World-systems analysis: An introduction. Duke University Press.

Wunder, S. 2005. Payments for environmental services: some nuts and bolts. CIFOR Occasional Paper No. 42. Pp. 24.

Wunder, S. 2013. When payments for environmental services will work for conservation. Conservation Letters 6 : 230-237.

Wunder, S. 2015. Revisiting the concept of payments for environmental services. Ecological Economics 117:234-243.

Wunder, S., S. Engel, and S. Pagiola. 2008. Taking stock: A comparative analysis of payments for environmental services programs in developed and developing countries. Ecological Economics 65:834-852. 